Cite this: DOI: 10.1039/c8nr09728c

\title{
Graphene hybrid materials: the role of graphene materials in the final structure of hydrogels $\uparrow$
}

\author{
Cristina Martín,,$^{a}$ Ana Martín-Pacheco, $\hbar^{a}$ Alicia Naranjo, ${ }^{a}$ Alejandro Criado, (DD ${ }^{b}$ \\ Sonia Merino, ${ }^{a, c}$ Enrique Díez-Barra, (D) a,c María Antonia Herrero (DD ${ }^{a, c}$ and \\ Ester Vázquez (iD) *a,c
}

Graphene (G), graphene oxide (GO) and graphene quantum dots (GQDs) have been introduced into a three-dimensional polymeric network based on polyacrylamide in order to ascertain the role of each nanomaterial in hydrogels. The hydrogel structure is not affected by the introduction of GQDs, since these nanoparticles do not form part of the polymeric network. $G$ and GO modify the structure of the hydrogels but in a different way. GO seems to interact by hydrogen bonding to form non-homogeneous gels in which the mechanical properties are not markedly improved. However, G takes an active part in the formation of the polymeric network, which leads to improved mechanical properties and stability of

the final material to give rise to truly hybrid hydrogels and not mere two-phase composite materials.

DOI: $10.1039 / c 8 n r 09728 c$

rsc.li/nanoscale

\section{Introduction}

${ }^{a}$ Instituto Regional de Investigación Cientifica Aplicada (IRICA), 13071 Ciudad Real, Spain

${ }^{b}$ CIC BiomaGUNE, Parque Tecnológico de San Sebastián, Paseo Miramón, 182, 20014 San Sebastián, Guipúzcoa, Spain

${ }^{c}$ Departamento de Quimica Orgánica, Facultad de Ciencias y Tecnologías Quimicas, Universidad de Castilla-La Mancha (UCLM), 713071 Ciudad Real, Spain.

E-mail: ester.vazquez@uclm.es

$\dagger$ Electronic supplementary information (ESI) available. See DOI: 10.1039/ c8nr09728c

$\ddagger$ These authors contributed equally to this work. and functional properties of the polymeric network has a crucial role in the use of the final material. Indeed, stimuliresponsive hydrogels ${ }^{7}$ or self-healing hydrogels ${ }^{8}$ prepared by using carbon nanomaterials have been the focus of intensive research in recent years due to their applications in different fields. ${ }^{9,10}$

Graphene $(\mathrm{G})$ is a two-dimensional nanomaterial in which the carbon atoms condense in a honeycomb lattice due to their $\mathrm{sp}^{2}$ hybridization. This pattern imparts the material with many extraordinary features, such as flexibility, conductivity and strength. ${ }^{11,12}$ Graphene Oxide (GO), by contrast, is an oxidized form of graphene decorated with multiple oxygen groups. ${ }^{13}$ The presence of these functional groups on graphene sheets modifies the nanomaterial structure and properties. G and GO therefore have very different physicochemical properties. ${ }^{14}$ In fact, GO is an insulating material. Finally, Graphene Quantum Dots (GQDs) are nanometre-sized carbon nanomaterials that have photoluminescence properties due to the quantum confinement and the edge effects. GQDs are only several nanometres in size and they may also have some functional groups at the edges. ${ }^{15}$

Due to their exceptional properties, CNM-based hydrogels have received attention from a large number of scientists. Regarding chemical hydrogels based on graphene derivatives, researchers indicate an enhancement of different hydrogel properties when these materials are incorporated into the network. For instance, Fan et al. improved the tenacity of a hydrogel based on polyacrylic acid by adding a graphene material obtained by exfoliation of graphite mediated by glycidyl methacrylate and gum arabic. ${ }^{16}$ Moreover, Servant 
et al. introduced $N, N^{\prime}$-methylenebisacrylamide (MBA) as a cross-linker in the methacrylic acid polymerization and this afforded a hydrogel with a high swelling degree and the ability to incorporate graphene flakes dispersed in water; the hydrogel structure was maintained and the electrical properties were enhanced. ${ }^{17}$ However, the role of graphene materials in hydrogel structures has only been reported in a few papers. Das et al. reported the in situ polymerization of acrylamide in a graphene dispersion and proposed a cross-linker function of graphene. ${ }^{18}$ Furthermore, in the previous work we studied the introduction of graphene as an intrinsic component in a polyacrylamide-based hydrogel. ${ }^{19}$

GO and reduced graphene oxide (rGO) have also been incorporated into chemical hydrogels. In most cases, GO or rGO are believed to act as cross-linkers through the functional groups attached to their surface. As an example, Zhang et al. reported a GO-clay- $N$-isopropylacrylamide (PNIPAm) hydrogel in which the nanomaterial interacts by hydrogen bonding with PNIPAm. ${ }^{20}$ Bai et al. reported a graphene oxide/polyvinyl alcohol (PVA) pH-sensitive hybrid for drug delivery. In this case, GO acts as a cross-linker due to the hydrogen bonds formed between its oxygen-based functional groups and the PVA. ${ }^{21}$ GO also acts as a cross-linker in the polyacrylamide hybrids reported by Zhang et al. ${ }^{22}$ Furthermore, a ternary hydrogel containing GO, as a cross-linker via hydrogen interactions, sodium alginate and polyacrylamide was reported by Fan et al. and this material showed improved elasticity and compressive strength. ${ }^{23} \mathrm{Hu}$ et al. thermally incorporated rGO and this improved the catalytic activity of a polyacrylamide-Agbased hydrogel. $\mathrm{Hu}$ et al. proposed a cross-linking network formed by strong physical interactions between the functional groups of the polymer chain and the oxygen functionalities on the graphene surface. ${ }^{24}$ In addition, GO was functionalized and linked covalently with a modified hyaluronic acid in the hydrogel reported by Byun and Lee. ${ }^{25}$

In the case of GQDs, and to the best of our knowledge, most of the examples described in the literature incorporate this graphene derivative in physical hydrogels. ${ }^{26}$ However, there are some cases in which carbon or graphene quantum dots are incorporated into chemically cross-linked hydrogels. For example, functionalized carbon dots have been incorporated as cross-linkers in chemical hydrogels. In this respect, a 2-hydroxyethyl methacrylate (HEMA) and acrylic acid hydrogel based on HEMA-modified carbon dots as chemical crosslinkers has been prepared. ${ }^{27}$ The incorporation of the HEMAcarbon dots was crucial for the luminescence properties of the material and for the improvement of the mechanical strength. Moreover, a chemically cross-linked hydrogel based on acrylamide and acrylic acid was prepared as a white-light-emitting hydrogel. ${ }^{28}$ Functionalized carbon dots were incorporated into the network by polymer coupling due to the presence of acrylic groups on the surface. This material had good mechanical properties, which were enhanced when carbon dots were added. Regarding GQDs, Zhou et al. described how GQDs can be used to form fluorescent acrylamide-based hydrogels. ${ }^{29}$ Nevertheless, the way in which the GQDs were retained in the network was not explained, and the characterization of the composite was not described.

With the aim of comparing and verifying the role of carbon nanomaterials on the structure and features of a three-dimensional (3D) polymeric network, we selected three graphene materials with different physicochemical characteristics: G, GO and GQDs. In order to avoid misunderstanding and misinterpretation of the different studies, as the first step we describe the features of the nanomaterial used to create a nanocomposite hydrogel.

There are still some issues regarding the standardization of the specific physicochemical characteristics of 2D carbon materials,$^{30,31}$ and it was decided here to characterize these three materials according to their size and chemical functional groups. G and GO have comparable sizes, while GQDs are at least two orders of magnitude smaller. On the other hand, the surface of GQDs is chemically more similar to that of GO. Finally, these three materials were employed for the preparation and characterization of the features of diverse final nanocomposite structures based on polyacrylamide. The content of nanomaterials in the final hydrogels was similar in the three cases and comparable to the one used in our previous studies (nanomaterial:acrylamide ratio ranging from 0.001 to 0.1$).{ }^{17,19}$ This ratio is on the same order as that of the amount of cross-linker, being much lower than the nanomaterial:monomer ratio employed in other studies (Table S1 $\uparrow$ ).

\section{Experimental}

\section{Materials}

Reagents were used as purchased from commercial sources without further purification. SP-1 graphite powder was purchased from Bay Carbon, Inc. Melamine, sodium percarbonate (SPC), acrylamide, $N, N^{\prime}$-methylenebisacrylamide (MBA) and potassium persulfate (KPS) were purchased from Sigma Aldrich. A planetary ball mill (Restch PM 100) was used for the preparation of $\mathrm{G}$ and GQDs.

\section{Preparation of the aqueous G dispersions}

The exfoliation of graphite was carried out using a ball-milling procedure. ${ }^{32,33} 30 \mathrm{mg}$ of a graphite/melamine mixture $(1: 3)$ was ball-milled at $100 \mathrm{rpm}$ for 30 minutes. The resulting solid mixture was transferred into an Erlenmeyer flask using $20 \mathrm{~mL}$ of deionized water and ultrasonication led to a dark suspension. Melamine was removed by washing the dispersion with hot water $\left(70{ }^{\circ} \mathrm{C}\right)$. Dialysis against deionized water through a dialysis membrane (MWCO: 6-8 kDa) was used for this purpose. During the dialysis, the permeate was removed every 2 hours at least 5 times and overnight. The obtained suspension was left to settle for five days and the unreacted graphite was removed. The liquid fraction with stable sheets in suspension was carefully extracted to provide few-layer graphene. 


\section{Preparation of the aqueous GO dispersions}

GO flakes provided by Grupo Antolín Ingeniería ${ }^{34}$ were washed by centrifugation. $40 \mathrm{mg}$ of $\mathrm{GO}$ and $40 \mathrm{~mL}$ of deionized water were centrifuged for $30 \mathrm{~min}$ at $4000 \mathrm{rpm}$, controlling the $\mathrm{pH}$ of the medium. The procedure was repeated until the $\mathrm{pH}$ remained at approximately 5 .

\section{Preparation of the aqueous GQD solutions}

10 GQD solutions were prepared following a mechanochemical treatment. ${ }^{35} 200 \mathrm{mg}$ of graphite and $100 \mathrm{mg}$ of sodium percarbonate (SPC) were ball-milled for twelve hours at $400 \mathrm{rpm}$. The resulting powder was collected with deionized water $(2 \mathrm{~L}$ approximately) in an Erlenmeyer flask and filtered using a $0.2 \mu \mathrm{m}$ pore Millipore filter. The filtrate was concentrated on a rotary evaporator and dialyzed against deionized water though a dialysis membrane (MWCO: $3500 \mathrm{Da}$ ) for 24 hours in order to remove unreacted SPC. During the dialysis, the permeate was removed every 2 hours and the dialysis was continued overnight. Finally, to obtain the powder the solvent was removed on a rotary evaporator.

\section{Characterization of nanomaterials}

A thermogravimetric analysis equipment Q50 from TA Instruments was used to perform thermogravimetric analyses (TGA) at $10^{\circ} \mathrm{C} \mathrm{min}^{-1}$ under a nitrogen atmosphere.

An inVia Renishaw microspectrometer $(532 \mathrm{~nm}$ point-based laser) was used to record Raman spectra. Raman samples were prepared by dropping G, GO and GQD dispersions onto silicon oxide surfaces (provided by WRS Materials). The obtained spectra of G, GO and GQDs were treated according to baseline correction, noise filtering and normalization. Moreover, in the case of G, they were fitted with Lorentzian-shaped bands to ascertain band positions, widths and intensities.

Transmission Electron Microscopy (TEM) and High Resolution TEM (HRTEM) were carried out on a Jeol 2100 HRTEM system. The samples were prepared by immersion of the nickel grid (LC200-Ni Lacey Carbon Grid from Electron Microscopy Sciences) into the G, GO and GQDs dispersions.

Q5 A spectrofluorometer from Photon Technology International Inc. was used to record photoluminescence (PL) spectra, using a slit width of $1 \mathrm{~nm}$ and a quartz cuvette with a $10 \mathrm{~mm}$ path length.

X-Ray Photoelectron Spectroscopy (XPS) was performed on a SPECS Sage HR 100 spectrometer with a nonmonochromatic aluminium-based X-ray source with a $\mathrm{K} \alpha$ line of $1486.6 \mathrm{eV}$ energy and $300 \mathrm{~W}$.

\section{Preparation of the blank polyacrylamide hydrogels}

Blank polyacrylamide hydrogels were prepared using acrylamide as the main monomer, MBA as the cross-linker and KPS as the initiator. ${ }^{19}$ Acrylamide and MBA were dissolved in water (200 $\mathrm{mg} \mathrm{mL}^{-1}$ and $0.2 \mathrm{mg} \mathrm{mL} \mathrm{mL}^{-1}$, respectively). The solution was homogenized by stirring and mild sonication. The initiator was added $\left(0.4 \mathrm{mg} \mathrm{mL} \mathrm{m}^{-1}\right)$. Polymerization took place at $75{ }^{\circ} \mathrm{C}$ for 1 hour. Once the hydrogel was synthesized, the scaffold was submerged in a $600 \mathrm{~mL}$ beaker that contains $500 \mathrm{~mL}$ of water at room temperature $(\sim 0.05 \mathrm{~g}$ of the final freshly prepared material per $\mathrm{mL}$ of water). The water was replaced every day for four days in order to remove any unreacted small molecules, following the protocol used in other hydrogel related studies. ${ }^{17,19}$ The water from the washing cycles was evaporated and no appreciable residue was obtained, meaning that the reaction was efficient and the amount of unreacted chemicals was negligible. Once washed, the blank hydrogel could be dried in an oven to give the respective xerogel.

\section{Preparation of nanomaterial-based polyacrylamide hydrogels}

A similar process to that described above for the preparation of blank polyacrylamide hydrogels was followed to synthesize the nanocomposites in situ, adding in this case all of the reactants to the aqueous nanomaterial dispersion. G, GO and GQD water dispersions with different concentrations were used $\left(0.2 \mathrm{mg} \mathrm{mL} \mathrm{m}^{-1}\right.$ and $2 \mathrm{mg} \mathrm{mL} \mathrm{m}^{-1}$ ). In the case of the absorbed samples, xerogels were immersed in $20 \mathrm{~mL}$ of the aqueous nanomaterial dispersion $\left(0.05 \mathrm{mg} \mathrm{mL}^{-1}\right)$ for 1 day instead of using them as a reaction solvent.

\section{Swelling studies}

Swelling studies were performed in lyophilized hydrogel samples after bringing them to the maximum volume degree. The resulting aerogels were immersed in ultrapure deionized water at room temperature. All of the samples had a similar volume $\left(\approx 0.5 \mathrm{~cm}^{3}\right)$. The weights of the swollen hydrogels were recorded at known time intervals until they reached a constant weight. Excess water on the surface of the gels was removed with filter paper before weighing. The swelling degree was then calculated.

\section{Scanning electron microscopy (SEM) experiments}

Blank and nanocomposite hydrogels were completely swollen, frozen, and then dried overnight in a Telstar Lyoquest freezedrier to yield dry aerogel samples. The hydrogel microstructure was analysed by SEM using an FEI QUANTA 250 system. The distribution of the pore size was measured for all samples from the SEM images using Fiji® software.

\section{Mechanical properties}

Compressive and tensile tests were carried out on samples at $25{ }^{\circ} \mathrm{C}$ with a Mecmesin Multitest 2.5-i dynamic mechanical analyser. For compressive studies, cylindrical disks of hydrogels (×6) with a diameter of $1.5 \mathrm{~cm}$ and an initial thickness of $1.5 \mathrm{~cm}$ were molded. Hydrogel disks were uniaxially compressed between two plates $\left(6 \mathrm{~mm} \mathrm{~min}^{-1}\right.$ and cell load $\left.=50 \mathrm{~N}\right)$. The extent of the deformation was measured in terms of strain (70\%). Young's modulus values were calculated between $15 \%$ and $20 \%$ of strain. The absorbed energy values were calculated between $0 \%$ and $20 \%$ of strain. A mold made of Teflon which meets the ISO 37 standard was used to prepare the samples for tensile tests. Hydrogel specimens $(\times 6)$ were uniaxially stretched until breaking $\left(50 \mathrm{~mm} \mathrm{~min}^{-1}\right.$ and cell load $\left.=50 \mathrm{~N}\right)$. 
$1 \quad$ Results and discussion

Preparation and characterization of the carbon nanomaterials

$\mathrm{G}$ flakes were prepared by mechanochemical exfoliation of graphite though interactions with melamine (2,4,6-triamine1,3,5-triazine) under solvent-free conditions using a ballmilling treatment. ${ }^{32,33}$ Briefly, $22.5 \mathrm{mg}$ of graphite powder and $7.5 \mathrm{mg}$ of melamine were milled under dry conditions, and the exfoliation was achieved at $100 \mathrm{rpm}$ in only 30 minutes. Once milled, the melamine was removed by dialysis against water, and the nonreactive graphite was separated by natural sedimentation to leave few-layer graphene in stable black suspensions with a final concentration of $0.12 \mathrm{mg} \mathrm{mL}^{-1}$ in deionized water (see the Experimental section for more details). The graphene material in the water dispersions was thoroughly characterized (Fig. 1). TGA showed a weight loss of $9 \%$ at $600{ }^{\circ} \mathrm{C}$, which corresponds to a low concentration of oxygen groups. The Raman spectrum of $\mathrm{G}$ contained the two most intense peaks of this CNM: the G peak at around $1580 \mathrm{~cm}^{-1}$ and the $2 \mathrm{D}$ band at around $2700 \mathrm{~cm}^{-1} \cdot{ }^{36} \mathrm{D}^{\prime}$ appeared as a shoulder on the $\mathrm{G}$ band at around $1600 \mathrm{~cm}^{-1}$. In addition, the FWHM (2D) was 60.6 and $I(2 \mathrm{D}) / I(\mathrm{G})$ was 0.45 , thus confirming that the sample was few-layer graphene. ${ }^{36}$ In fact, the number of layers was calculated according to Paton et al. ${ }^{37}$ and a value of 4 layers was determined. Finally, the Raman feature at $1345 \mathrm{~cm}^{-1}$ (D band) was observed in the spectrum. The average $I(\mathrm{D}) / I(\mathrm{G})$ was $0.40 \pm 0.08$, which is attributed to the defects at the edges of the sub-micrometre flakes. ${ }^{32}$ XPS was performed to analyse the low concentration of functional groups. The $\mathrm{C}$ 1s core-level spectra indicate the existence of four different bonds: the most intense peak, at $284.5 \mathrm{eV}$, corresponds to $\mathrm{sp}^{2} \mathrm{C}$-atoms. This peak, together with the one at $290.9 \mathrm{eV}$ from $\pi-\pi$ transitions, provides evidence for the graphitic carbon structure. Moreover, peaks at 286.5 and $288.7 \mathrm{eV}$ from $\mathrm{C}-\mathrm{O}$ and $\mathrm{O}-\mathrm{C}=\mathrm{O}$ bonds only appear with a very low intensity (Fig. S1a †). A size distribution around 200-2000 nm was found by TEM (Fig. 1). A representative image of $G$ is shown in Fig. S2a. $\dagger$

GO powder was washed in order to guarantee the complete removal of acids. Therefore, several washing cycles were performed by centrifugation using deionized water until a $\mathrm{pH} \approx 5$ was obtained in the GO dispersion (see the Experimental section for more details). The resulting GO was completely characterized (Fig. 1). TGA gave evidence for the thermal instability of the GO sample. A weight loss of $46 \%$ was observed at $600{ }^{\circ} \mathrm{C}$ due to a high number of oxygenated groups within the structure. The Raman spectrum contained the typical D and $\mathrm{G}$ bands at $1345 \mathrm{~cm}^{-1}$ and $1580 \mathrm{~cm}^{-1}$, respectively. An $I(\mathrm{D}) / I(\mathrm{G})$ ratio of 0.96 and a low $2 \mathrm{D}$ band intensity were observed, ${ }^{38}$ thus confirming the presence of a high concentration of defects. The $\mathrm{C} 1 \mathrm{~s}$ core-level spectra from XPS could be deconvoluted into five different bonds (Fig. S1b†). In the case of GO, and in contrast to G, the most intense peak, at $286.6 \mathrm{eV}$, corresponds to the $\mathrm{C}-\mathrm{O}$ bond. Moreover, peaks at 288.4 and $290.1 \mathrm{eV}$ (from $\mathrm{C}=\mathrm{O}$ and $\mathrm{O}-\mathrm{C}=\mathrm{O}$ species, respectively) indicate a larger amount of functional groups. The peak

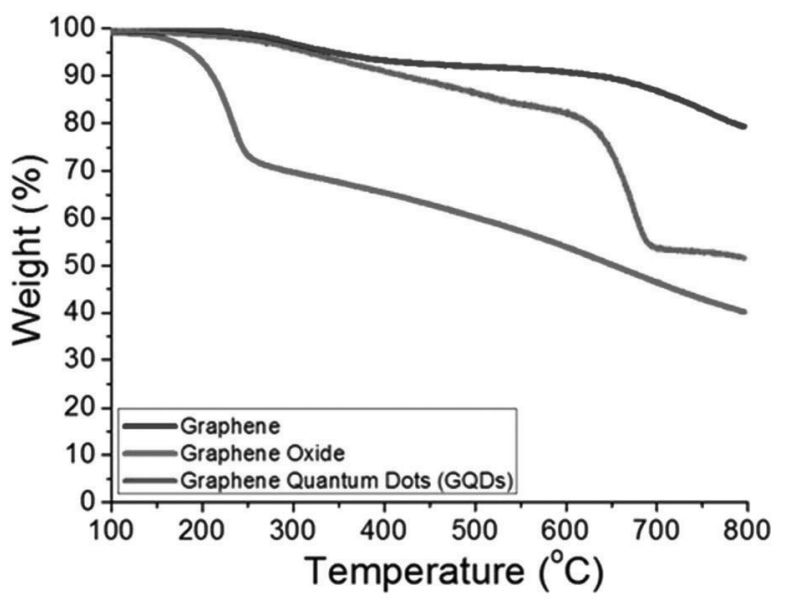

1

10

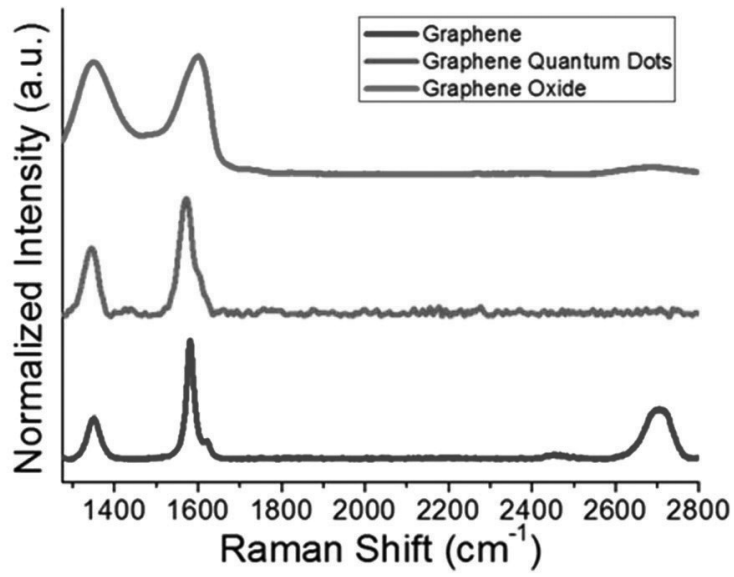

20

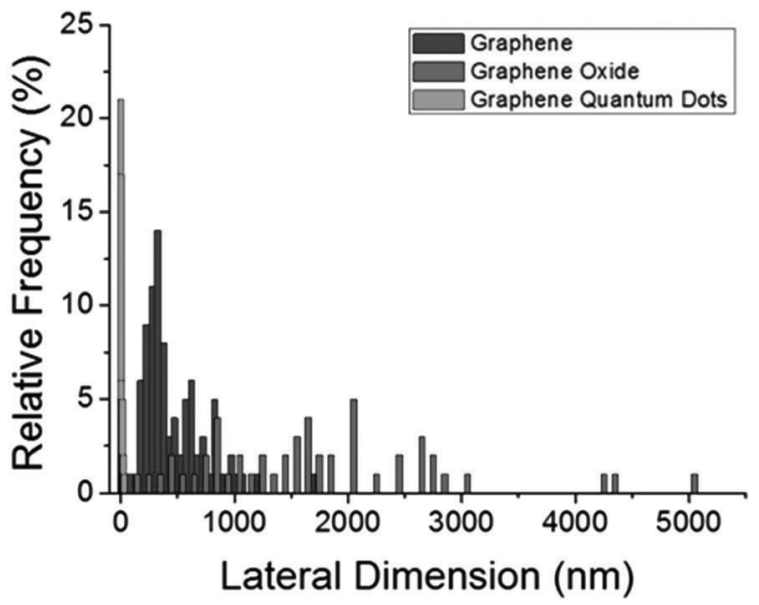

35

Fig. 1 Characterization of G (blue), GO (red) and GQDs (green). From top to bottom: thermogravimetric analysis, average Raman spectrum (at least 18 spectra from different sample locations) and lateral size distribution by TEM.

at 284.5 from $\mathrm{C}-\mathrm{C}$ is due to the graphitic structure. TEM revealed a lateral dimension that ranged from 150 to $5000 \mathrm{~nm}$ (Fig. 1). A representative image of GO is provided in Fig. S2b. $\dagger$ 
GQDs were prepared by ball-milling of graphite in the presence of SPC as a solid oxidant. ${ }^{35}$ The resulting material was dispersed in water and then purified. Both filtration through a $0.2 \mu \mathrm{m}$ pore filter and dialysis through a $3500 \mathrm{Da}$ membrane were employed to obtain the purified GQDs (see the Experimental section for more details). The resulting GQDs were highly soluble in water and they had photoluminescence properties. The highest PL intensity was observed at $430 \mathrm{~nm}$ using an excitation wavelength of $315 \mathrm{~nm}$. The GQDs were thoroughly characterized (Fig. 1). TGA showed a weight loss of $18 \%$ at $600{ }^{\circ} \mathrm{C}$ due to the presence of oxygen groups on the GQD structure. The Raman spectrum showed $\mathrm{D}$ and $\mathrm{G}$ bands with an intensity ratio $I(\mathrm{D}) / I(\mathrm{G})$ of 0.6 . The absence of the $2 \mathrm{D}$ band indicates disruption of the stacking order, thus confirming the presence of oxygen groups. $^{38}$ This fact was corroborated by XPS, which provided evidence for the presence of five different bonds, i.e., $\mathrm{C}-\mathrm{O}(286.2 \mathrm{eV}), \quad \mathrm{C}=\mathrm{O} \quad(287.3 \mathrm{eV}), \mathrm{COOH}$ $(288.6 \mathrm{eV})$, and $\mathrm{COO}^{-}(290.4 \mathrm{eV})$, with the most intense peak at $284.8 \mathrm{eV}$ corresponding to $\mathrm{C}-\mathrm{C}$ bonds (Fig. S1c $\dagger$ ). The HRTEM histogram revealed a lateral dimension that rises from 1.5 to $20 \mathrm{~nm}$ (Fig. 1). A representative image of the GQDs is given in Fig. S2c. $\dagger$

The three products described above were used to study the role of different carbon nanomaterials in the polyacrylamide network depending on their physicochemical properties: $\mathrm{G}$ is comparable to GO in terms of the lateral dimensions but it has completely different chemical surfaces. On the other hand, GO is comparable to GQDs with respect to the functional groups on their surfaces, but they have totally different sheet sizes (Fig. 1 and $\mathrm{S} 1 \dagger$ ).
Preparation and characterization of hydrogels

Polyacrylamide hydrogels were prepared by polymerization of acrylamide using MBA as a cross-linker and KPS as an initiator. ${ }^{19}$ For the preparation of the blank hydrogel both amides were dissolved in water and the solution was homogenized by stirring and sonication. The initiator was then added and the polymerization took place for one hour. To prepare the in situ hydrogels based on different nanomaterials, the amides were dissolved in the respective nanomaterial aqueous dispersions (G, GO and GQDs) and the polymerization process was performed (Scheme S1a $\dagger$ ).

All of the final hydrogel structures were immersed in water for at least four days and the water was replaced every day to ensure the removal of any unreacted monomer, cross-linker or initiator. Finally, the washed hydrogels were dried by heating and xerogels were therefore obtained.

The microstructures of the in situ hydrogels based on G, GO and GQDs were analysed by SEM (Fig. 2). For both G- and GObased hydrogels, the nanomaterial concentration was $0.2(0.2-$ AMG and 0.2-AMGO, respectively) or $2 \mathrm{mg} \mathrm{mL}^{-1}$ (2-AMG and 2-AMGO, respectively), while for the GQD-based hydrogel the concentration was $0.2 \mathrm{mg} \mathrm{mL}^{-1}$ (AMGQDs). As observed in previous studies, ${ }^{19}$ an increase in the $\mathrm{G}$ content leads to a reduction in the pore size. Therefore, 0.2-AMG and 2-AMG had a smaller pore size $(59 \pm 10$ and $53 \pm 19 \mu \mathrm{m}$, respectively) (Fig. 2c and d) in comparison with the average pore size in the blank hydrogel $(441 \pm 57 \mu \mathrm{m})$ (Fig. 2a). In the case of GO-based hydrogels, a heterogeneous pore morphology was observed in both cases, 0.2-AMGO and 2-AMGO, with comparable pore sizes (around $27 \mu \mathrm{m}$ ), but different pore structures (Fig. 2e and
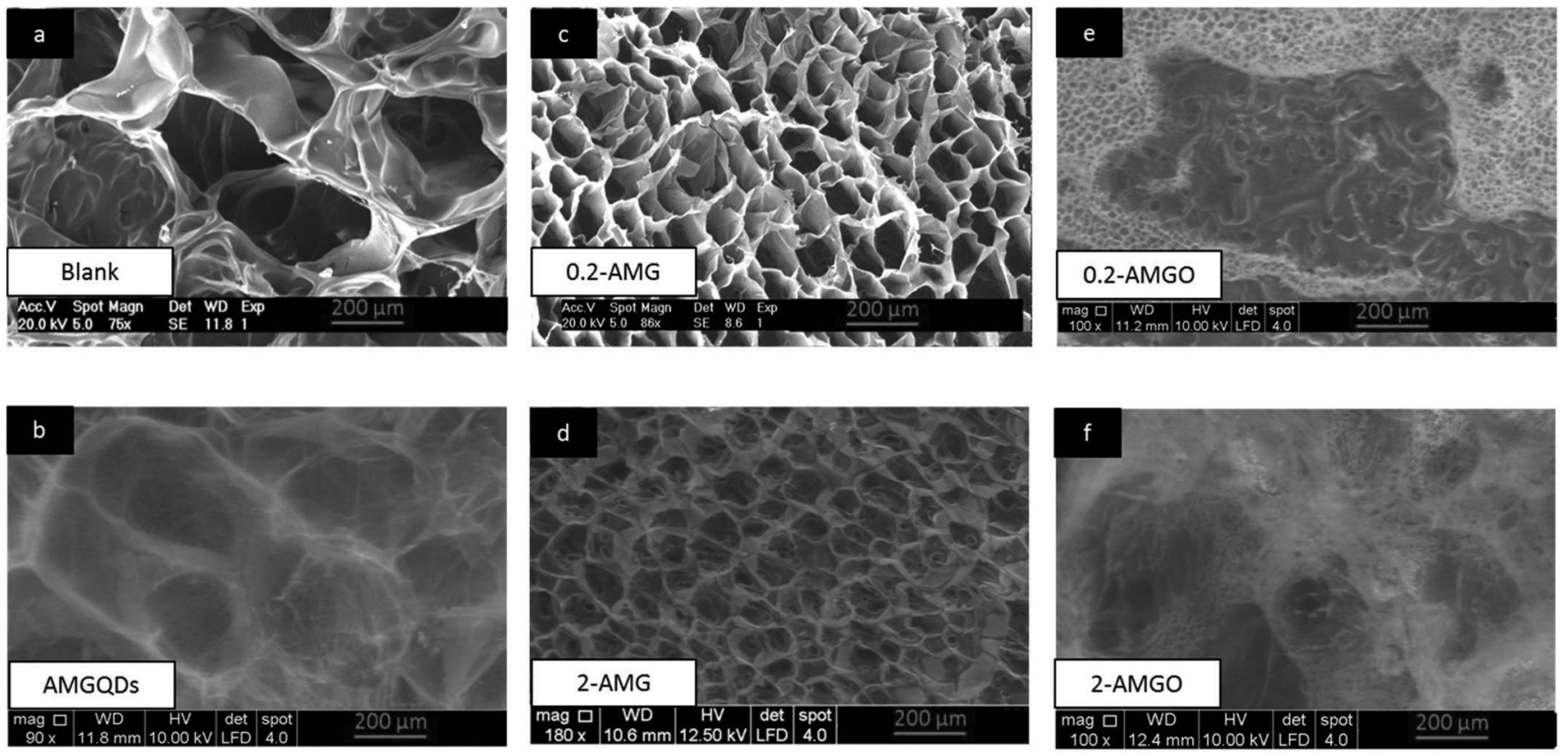

Fig. 2 SEM images of (a) blank hydrogel; (b) in situ GQD-based hydrogels at $0.2 \mathrm{mg} \mathrm{mL}^{-1}$; (c, d) in situ G-based hydrogels at 0.2 and $2 \mathrm{mg} \mathrm{mL}^{-1}$, respectively; $(\mathrm{e}, \mathrm{f})$ in situ GO-based hydrogels at 0.2 and $2 \mathrm{mg} \mathrm{mL}^{-1}$, respectively. 
f, and Fig. S3†). The decrease in the pore size in the presence of both nanomaterials suggests that $\mathrm{G}$ and GO could play a role in the polymer network. As reported previously, ${ }^{20-23} \mathrm{GO}$ can act as a cross-linker by forming hydrogen bonds with the functional groups of the polymeric chains. However, considering the low amount of oxygen groups on the G surface, such interactions are hard to envisage. Finally, regarding the pore size in GQD-based hydrogels, the value is $518 \pm 139 \mu \mathrm{m}$, which is quite similar to that determined for the blank hydrogels (Fig. 2b). This result suggests that GQDs do not form part of the polymer network. In order to confirm this situation, the water from washing cycles of GQD-based hydrogels was analysed by PL spectroscopy. It can be seen from Fig. 3 and Fig. S4 $\uparrow$ that, compared to the blank hydrogel, the fluorescence intensity of the initial GQD-based hydrogel progressively decreased after each washing cycle and the washing water became fluorescent (Fig. S5†). This result clearly indicates that GQDs are not involved in the 3D polymer structure since they are not retained within the hydrogel. The SEM images of GQDbased hydrogels before the washing cycles and after removal of the GQDs due to these cycles show a similar pore size and morphology, which corroborates the idea of poor interactions between the nanomaterial and the polymeric network (Fig. S6 $†$ ). In a previous study, we demonstrated the incorporation of GQDs into a different hydrogel, which became fluorescent due to the presence of the nanomaterial. ${ }^{35}$ The incorporation of GQDs was possible through electrostatic interactions with the network. In the case of polyacrylamide, a cross-linking role of GQDs due to hydrogen bonding, as described for GO, could also be expected. However, the GQDs are significantly smaller than GO sheets (Fig. 1) and XPS of GO (Fig. S1 $\dagger$ ) evidenced a higher amount of functional groups compared to GQDs. ${ }^{35}$ Therefore, these interactions could occur with GO but they could be negligible for GQDs. In conclusion, it can be considered that GQDs do not take part in the final polyacrylamide hydrogel structure.

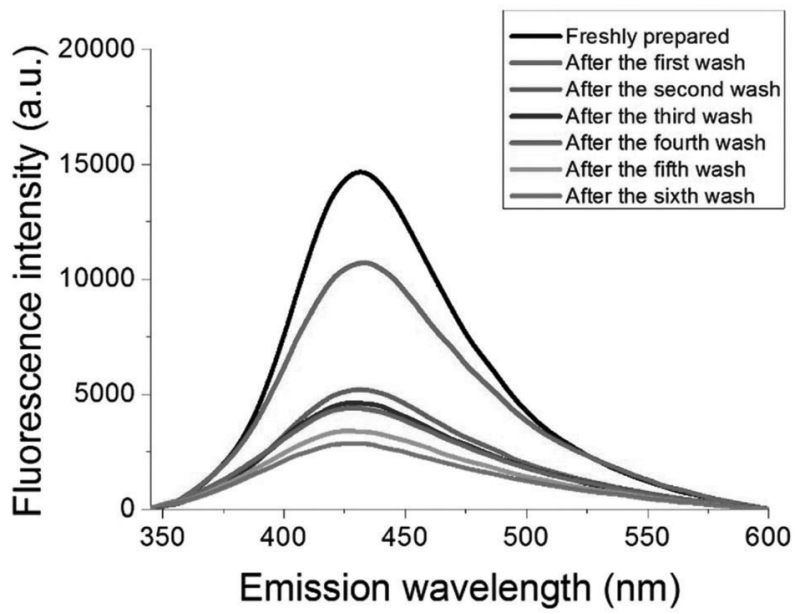

Fig. 3 Fluorescence spectra of the freshly prepared GQD-based hydrogel and after washing cycles.
A TGA study of the different hydrogels was carried out to confirm the results discussed above (Fig. S7 $\dagger$ ). It can be seen that only the blank hydrogel suffers a weight loss at $200{ }^{\circ} \mathrm{C}$ and this corresponds to the loss of $\mathrm{NH}_{3}$ from the primary amide groups; ${ }^{39}$ however, this loss is not observed in the case of the G-, GO- and GQD-based materials. This observation indicates that the incorporated CNMs interact to a certain degree with the amino groups of the polymeric network and help to stabilize it. The weight loss at around $280{ }^{\circ} \mathrm{C}$, which corresponds to the release of $\mathrm{CO}_{2}$, was observed at the same temperature for the blank sample and for AMGQDs, although a higher loss was observed in the latter gel as a consequence of the functional groups on the GQDs. Other changes were not observed in the TGA traces of the AMGQD hydrogels in comparison to the blank, thus confirming that further interactions do not take place between the polymeric matrix and the GQDs. Shifts to temperatures higher than $280^{\circ} \mathrm{C}$ were observed in the cases of AMG and AMGO hydrogels when compared to the blank sample, which is consistent with the idea of certain interactions between the NMs and the acrylamide network. Moreover, the weight loss trend was $\mathrm{AMG}<\mathrm{AMGO}$, i.e., in the same order as the presence of oxygenated groups in the respective NMs. Finally, the most intense weight loss - corresponding to the loss of aliphatic chains - was observed at around $370{ }^{\circ} \mathrm{C}$ in the case of AMGO hydrogels and this is the same temperature as the blank sample. However, for the AMG hydrogel this loss occurred at a higher temperature by $10{ }^{\circ} \mathrm{C}$ and this indicates a more intense interaction between $\mathrm{G}$ and the aliphatic network, which is not observed for GO. The different physicochemical characteristics of the two nanomaterials could be related to this result. As mentioned above, hydrogen bonds could be expected between GO and the polyacrylamide network, but in the case of $\mathrm{G}$, in accordance with the characterization shown in Fig. 1 and $\mathrm{S} 1, \dagger$ a different mode of attachment, such as covalent cross-linking, could also be suggested.

To characterize further the role of the NMs in the hydrogel structure, the swelling degree of the in situ G- and GO-based hydrogels was determined. As shown in Fig. 4, the final swelling degree of the hydrogel decreased in the presence of $\mathrm{G}^{19}$

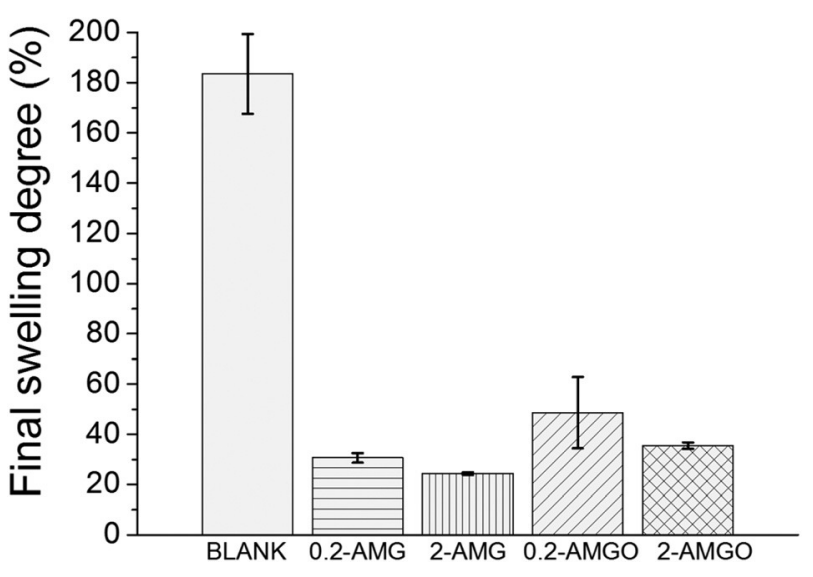


and GO. Furthermore, higher quantities of nanomaterial led to lower swelling degrees, thus confirming the interaction between the two nanomaterials and the polymer chains. The stronger hydrophobicity of $\mathrm{G}$ with respect to GO led to a lower swelling degree. Moreover, the mechanical properties of G- and GObased hydrogels were tested to evaluate the mechanical behaviour of both materials, which, in many cases, is known to be dependent on the pore distribution of the hydrogel.

The elasticity (Young's Modulus) of the hydrogels and the energy absorbed by these materials on applying stress were first estimated by compressive tests. Tensile experiments were also performed. In both cases, the influence of the nanomaterial concentration $\left(0.2\right.$ and $\left.2 \mathrm{mg} \mathrm{mL}^{-1}\right)$ on the mechanical properties was analysed (Fig. 5, 6, and S8 $\dagger$ ).

Compressive experiments showed that the Young's modulus of the blank hydrogel was not improved after the addition of $0.2 \mathrm{mg} \mathrm{mL}^{-1}$ of $\mathrm{G}$ and GO. However, these values changed upon increasing the concentration of G and GO to $2 \mathrm{mg} \mathrm{mL} \mathrm{m}^{-1}$. As can be observed in Fig. $5 \mathrm{a}$, in the case of the G-based hydrogel the Young's modulus increased, which suggests a stiffer material; nevertheless, in GO-based hydrogels, the Young's modulus decreased and this is indicative of poor elastic properties.

\section{a}
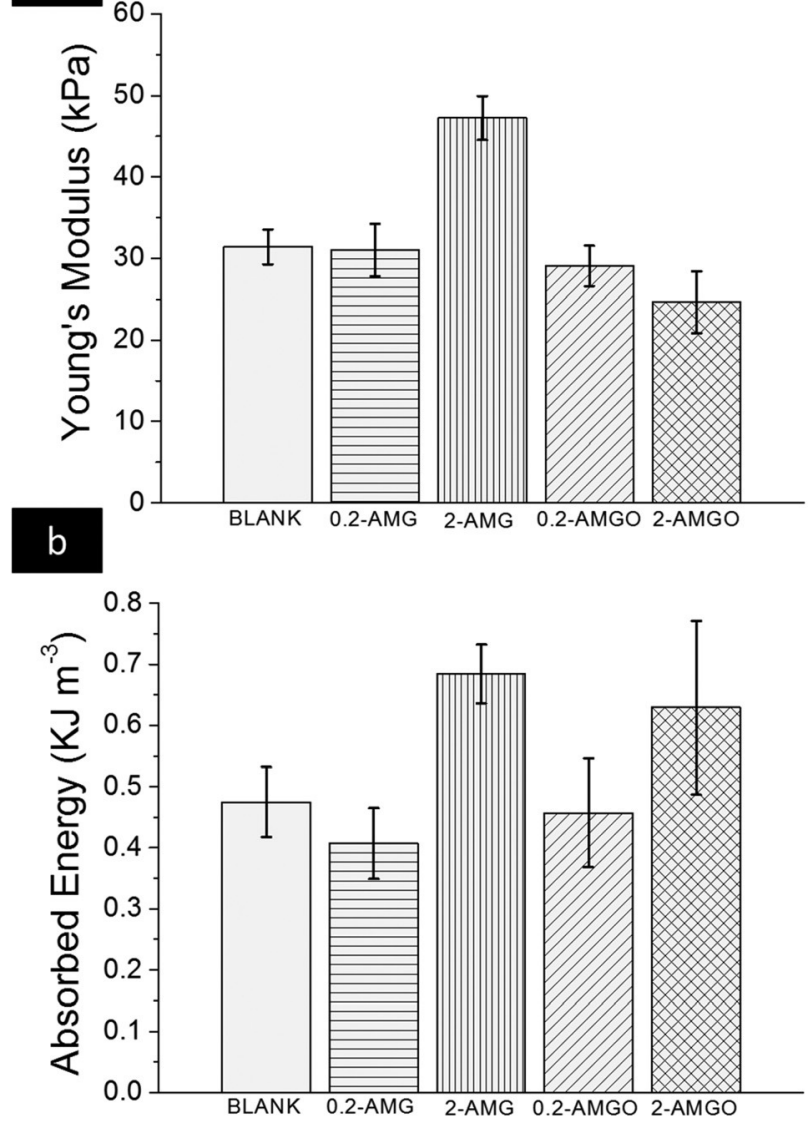

Fig. 5 (a) Young's modulus and (b) absorbed energy from the compressive test of hydrogel samples.

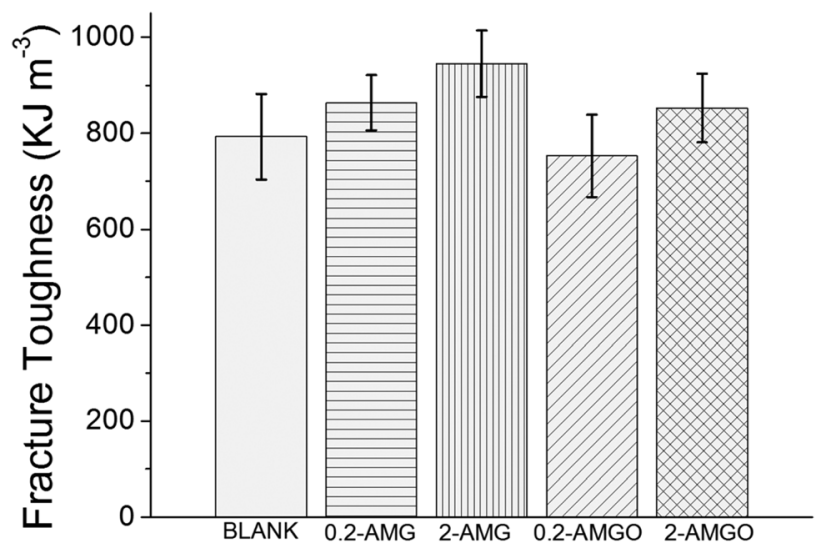

1

Fig. 6 Fracture toughness from tensile tests of hydrogel samples.

The energies absorbed by the hydrogels were determined by analysing the area under the stress/strain curves until only $20 \%$ of the strain was reached, since the dimensions of the samples remained unchanged in that linear range (Fig. 5b and $\mathrm{S} 8 \dagger$ ). G-based and GO-based hydrogels maintained similar values to those of the blank hydrogel when a low concentration

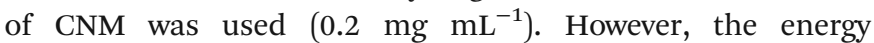
absorbed by the hydrogels increased on increasing the concentration of nanomaterial by a factor of $10\left(2 \mathrm{mg} \mathrm{mL}^{-1}\right)$. This finding highlights the important role of nanomaterials, in a certain concentration range, inside a polymer network regarding the energy absorbance capacity.

In addition to compressive studies, tensile experiments were carried out to test the materials until fracture. In this case, a mold made of Teflon, which meets the ISO 37 standard, was used to prepare the samples for tensile tests and the hydrogel specimens were uniaxially stretched until their breaking point.

It can be seen from Fig. 6 that the fracture toughness for 2-AMG is higher than that for the blank or for 0.2-AMG. Therefore, the sample with a concentration of $\mathrm{G}$ of $2 \mathrm{mg} \mathrm{mL}^{-1}$ absorbed more energy before breaking, thus confirming once again the intrinsic interaction between $\mathrm{G}$ and the polymeric network. In contrast, in the case of GO-based hydrogels significant differences in the fracture toughness were not observed in comparison with the blank.

The different results between G- and GO-based hydrogels could be caused in part by the lack of homogeneity of GObased samples. In fact, once the hydrogels had been swollen until their maximum swelling degree, it was possible to observe the GO aggregates with the naked eye (Fig. S9†). Nonhomogeneous GO-based hydrogels were also reported for methacrylic acid-based hydrogels. ${ }^{17}$

In an effort to evaluate in depth the kind of interaction between G or GO and the polymer network, the stability of the hydrogels was studied when the nanomaterial was added by absorption after polymerization of the blank hydrogel; these samples are denoted as absorbed samples (Scheme S1b†). Physical interactions (such as hydrogen bonds) should occur 
independently, regardless of whether graphene materials are present during the polymerization process or whether they are simply absorbed once the network has been formed. Therefore, comparison of the behaviour of the in situ samples and the absorbed samples under swelling/de-swelling cycles could provide additional information about the type of link between G or GO and polyacrylamide. All of the samples were subjected to three de-swelling/swelling cycles by heating them at $40{ }^{\circ} \mathrm{C}$ under vacuum $\left(9 \times 10^{-1} \mathrm{mbar}\right)$. The results were compared with those obtained on a sample of the blank hydrogel (without any nanomaterial), which was also tested as a control. This test was carried out by swelling in situ xerogels in $20 \mathrm{~mL}$ of water. In contrast, to prepare the absorbed hydrogels, blank xerogels were swollen in $20 \mathrm{~mL}$ of $\mathrm{G}$ or GO dispersions at $0.05 \mathrm{mg} \mathrm{mL}^{-1}$. Although the quantities of $\mathrm{G}$ and GO used were the same both in the in situ and in the absorbed materials ( $1 \mathrm{mg})$, the appearance of these samples was quite different. In the case of the absorbed hydrogels, $G$ was clearly located at the periphery of the hydrogel and very little was present in the bulk. Similar results were obtained for GO samples (Fig. 7).

Interestingly, the in situ samples with G and GO retained the nanomaterial inside after they were dried under vacuum; however, in the absorbed samples, G and GO were partially expelled and these materials were found to be scattered all around the flask walls after an identical vacuum operation (Fig. S10a and $\mathrm{b} \dagger$ ). This process of swelling and de-swelling under vacuum was repeated three times. After three cycles the G-based hydrogel obtained by in situ polymerization retained its structure (Fig. S10e $\dagger$ ). However, all of the other samples, including the in situ GO-based hydrogel, were completely broken due to their poor consistency (Fig. S10c, $d$ and $f \dagger$ ). The blank hydrogel only suffered slight deformations (Fig. S10g†).

This experiment clearly shows that physical interactions between G or GO and polyacrylamide chains are not sufficiently strong in their own right to retain the nanomaterial
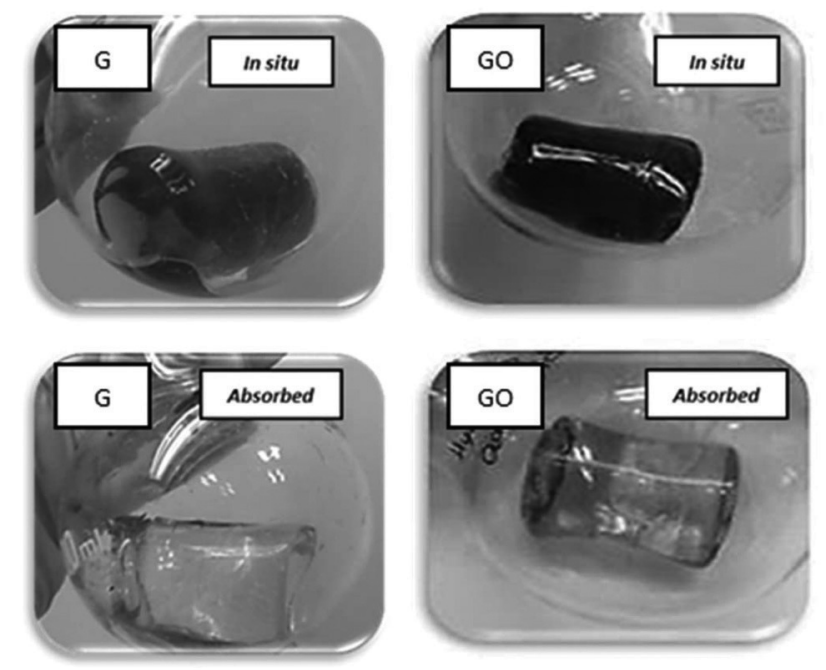

Fig. 7 Initial appearances of in situ and absorbed G- and GO-based hydrogels. inside the hydrogel. However, G-based hydrogels prepared by in situ polymerization showed quite different properties and behaviour compared to GO-based ones.

Therefore, one can conclude that G, in contrast to GO or GQDs, can be used as a covalent cross-linker in radical polymerization processes, possibly due to the higher number of active double bonds on its surface. ${ }^{40,41}$ This fact makes it possible to create novel functional hybrid hydrogels with new properties based on G. The ability of G to react with ethylenebased monomers, with different functional groups, in a covalent manner increases the range of applications of the final hybrid hydrogel.

\section{Conclusions}

The differences between the features of three hydrogels based on G, GO and GQDs were evaluated. The three carbon nanomaterials definitely interact differently with the polymer network. GQDs are not retained within the hydrogel when there are no electrostatic interactions between the components.

GO and G affect the morphology of the hydrogel, not only depending on the nature of the nanomaterial, but also on how it is incorporated into the polymer network. When carbon nanomaterials are absorbed into the network through a swelling process (absorbed), neither G nor GO are completely retained in the network after de-swelling. In contrast, if the nanomaterial is present when the polymerization takes place (in situ), both G and GO take part in the network, albeit in different ways.

The influence of GO and G on the mechanical properties of the hydrogels is also different. GO-based hydrogels are not homogeneous and the toughness of this material is lower than that of the blank hydrogel. On the other hand, G improves the elasticity of hydrogels (increase in Young's modulus) and, moreover, the behaviour of G-based hydrogels against fracture is improved. Furthermore, a higher concentration of G leads to better results. In conclusion, it was confirmed from the different nanomaterials that $\mathrm{G}$ is a unique component as it is incorporated as part of the polymeric network and therefore forms truly hybrid hydrogels and not mere two-phase composite materials.

\section{Conflicts of interest}

There are no conflicts to declare.

\section{Acknowledgements}

Financial support from the EU 785219-Graphene Core 2 European Union (Flagship project), the Spanish Ministerio de Economía y Competitividad (project CTQ2017-88158-R) and the FEDER-Junta de Comunidades de Castilla-La Mancha (project SBPLY/17/180501/000204 is gratefully 
acknowledged. A. Martín-Pacheco acknowledges the Spanish Ministerio de Educación, Cultura y Deporte for her grant (FPU14/01431).

\section{References}

1 S. Merino, C. Martín, K. Kostarelos, M. Prato and E. Vázquez, ACS Nano, 2015, 9, 4686-4697.

2 E. Caló and V. V. Khutoryanskiy, Eur. Polym. J., 2015, 65, 252-267.

3 J. Tavakoli and Y. Tang, Polymer, 2017, 9, 364.

4 E. M. Ahmed, J. Adv. Res., 2015, 6, 105-121.

5 H. Zhu, X. Cai, L. Wu and Z. Gu, J. Mater. Chem. B, 2017, 5, 3145-3153.

6 G. Hong, S. Diao, A. L. Antaris and H. Dai, Chem. Rev., 2015, 115, 10816-10906.

7 I. Willner, Acc. Chem. Res., 2017, 50, 657-658.

8 D. L. Taylor and M. in het Panhuis, Adv. Mater., 2016, 28, 9060-9093.

9 A. Vashist, A. Kaushik, A. Ghosal, J. Bala, R. NikkhahMoshaie, W. A. Wani, P. Manickam and M. Nair, Gels, 2018, 4, 75.

10 A. K. Gaharwar, N. A. Peppas and A. Khademhosseini, Biotechnol. Bioeng., 2014, 111, 441-453.

11 A. K. Geim, Science, 2009, 324, 1530-1534.

12 M. J. Allen, V. C. Tung and R. B. Kaner, Chem. Rev., 2010, 110, 132-145.

13 K. A. Mkhoyan, A. W. Contryman, J. Silcox, D. A. Stewart, G. Eda, C. Mattevi, S. Miller and M. Chhowalla, Nano Lett., 2009, 9, 1058-1063.

14 Y. Zhu, S. Murali, W. Cai, X. Li, J. W. Suk, J. R. Potts and R. S. Ruoff, Adv. Mater., 2010, 22, 3906-3924.

15 S. Zhu, Y. Song, X. Zhao, J. Shao, J. Zhang and B. Yang, Nano Res., 2015, 8, 355-381.

16 J. Fan, Z. Shi, J. Wang and J. Yin, Polym., 2013, 54, 39213930.

17 A. Servant, V. Leon, D. Jasim, L. Methven, P. Limousin, E. V. Fernandez-Pacheco, M. Prato and K. Kostarelos, Adv. Healthcare Mater., 2014, 3, 1334-1343.

18 S. Das, F. Irin, L. Ma, S. K. Bhattacharia, R. C. Hedden and M. J. Green, ACS Appl. Mater. Interfaces, 2013, 5, 8633-8640.

19 C. Martín, S. Merino, J. M. González-Domínguez, R. Rauti, L. Ballerini, M. Prato and E. Vázquez, Sci. Rep., 2017, 7, 10942.

20 E. Zhang, T. Wang, C. Lian, W. Sun, X. Liu and Z. Tong, Carbon, 2013, 62, 117-126. 46, 2376.

22 N. Zhang, R. Li, L. Zhang, H. Chen, W. Wang, Y. Liu, T. Wu, X. Wang, W. Wang, Y. Li, Y. Zhao and J. Gao, Soft Matter, 2011, 7, 7231.
23 J. Fan, Z. Shi, M. Lian, H. Li and J. Yin, J. Mater. Chem. A,

21 H. Bai, C. Li, X. Wang and G. Shi, Chem. Commun., 2010, 40 R. K. Layek and A. K. Nandi, Polymer, 2013, 54, 50872013, 1, 7433

24 H. Hu, J. H. Xin and H. Hu, J. Mater. Chem. A, 2014, 2, 11319-11333.

25 E. Byun and H. Lee, J. Nanosci. Nanotechnol., 2014, 14, 7395-7401.

26 S. Biswas, D. B. Rasale and A. K. Das, RSC Adv., 2016, 6, 54793-54800.

27 J. Wang, X. Ma, L. Wei, X. Zhu, Y. Zhu, G. Wang, T. Mei, J. Li and X. Wang, Colloid Polym. Sci., 2018, 296, 745-752.

28 Q. Zhu, L. Zhang, K. Van Vliet, A. Miserez and N. HoltenAndersen, ACS Appl. Mater. Interfaces, 2018, 10, 1040910418.

29 L. Zhou, F. Wu, J. Yu, Q. Deng, F. Zhang and G. Wang, Carbon, 2017, 118, 50-57.

30 A. Bianco, H.-M. Cheng, T. Enoki, Y. Gogotsi, R. H. Hurt, N. Koratkar, T. Kyotani, M. Monthioux, C. R. Park, J. M. D. Tascon and J. Zhang, Carbon, 2013, 65, 1-6.

31 P. Wick, A. E. Louw-Gaume, M. Kucki, H. F. Krug, K. Kostarelos, B. Fadeel, K. A. Dawson, A. Salvati, E. Vázquez, L. Ballerini, M. Tretiach, F. Benfenati, E. Flahaut, L. Gauthier, M. Prato and A. Bianco, Angew. Chem., Int. Ed., 2014, 53, 7714-7718.

32 V. León, J. M. González-Domínguez, J. L. G. Fierro, M. Prato and E. Vázquez, Nanoscale, 2016, 8, 14548-14555.

33 J. M. González-Domínguez, V. León, M. I. Lucío, M. Prato and E. Vázquez, Nat. Protoc., 2018, 13, 495-506.

$34 \mathrm{H}$. Varela-Rizo, I. Rodriguez-Pastor, C. Merino and I. Martin-Gullon, Carbon, 2010, 48, 3640-3643.

35 A. Martin Pacheco, A. E. Del Rio Castillo, C. Martín, M. A. Herrero, S. Merino, J. L. G. Fierro, E. Díez Barra and E. Vázquez, ACS Appl. Mater. Interfaces, 2018, 10, 1819218201.

36 U. Mogera, R. Dhanya, R. Pujar, C. Narayana and G. U. Kulkarni, J. Phys. Chem. Lett., 2015, 6, 4437-4443.

37 K. R. Paton, E. Varrla, C. Backes, R. J. Smith, U. Khan, A. O'Neill, C. Boland, M. Lotya, O. M. Istrate, P. King, T. Higgins, S. Barwich, P. May, P. Puczkarski, I. Ahmed, M. Moebius, H. Pettersson, E. Long, J. Coelho, S. E. O'Brien, E. K. McGuire, B. M. Sanchez, G. S. Duesberg, N. McEvoy, T. J. Pennycook, C. Downing, A. Crossley, V. Nicolosi and J. N. Coleman, Nat. Mater., 2014, 13, 624-630.

38 K. Krishnamoorthy, M. Veerapandian, K. Yun and S.-J. Kim, Carbon, 2013, 53, 38-49.

39 J. D. Van Dyke and K. L. Kasperski, J. Polym. Sci., Part A: Polym. Chem., 1993, 31, 1807-1823.

1 5103.

41 M.-C. Hsiao, S.-H. Liao, M.-Y. Yen, P.-I. Liu, N.-W. Pu, C.-A. Wang and C.-C. M. Ma, ACS Appl. Mater. Interfaces, 2010, 2, 3092-3099. 